\title{
SURFACE MODIFIED COALS FOR ENHANCED CATALYST DISPERSION AND LIQUEFACTION
}

Report for the Period

April 1, 1998 to September30, 1998

Dr. Yaw D. Yeboah

Principal Investigator

Department of Engineering

Clark Atlanta University

Atlanta, Georgia 30314

PREPARED FOR THE UNITED STATES DEPARTMENT OF ENERGY UNDER CONTRACT NUMBER DE-FG22-95PC95229 


\begin{abstract}
This progress report covers the six-month period April-September 1998 for the Department of Energy project DE-FG22-95PC95229 entitled, Surface Modified Coals for Enhanced Catalyst Dispersion and Liquefaction. The aim of the study is to enhance catalyst loading and dispersion in coal for improved liquefaction by preadsorption of surfactants and catalysts on to the coal. During this reporting period, zeta potential measurements were conducted to assess the surface charge on the raw, pretreated and catalyzed coal samples. The surface area, transmission spectroscopy and luminescence intensity of the raw coal and pretreated coal samples were also determined to assess the quality of the coal surface.
\end{abstract}

Across a broad range of $\mathrm{pH}$ values, the raw coal had an overall negative charge. Coal treated with anionic surfactant SDS maintained an overall net negative surface negative charge. The interaction between the coal and cationic surfactant DDAB caused the opposite effect resulting in a more positive coal surface charge. Although one would have expected little or no effect of the neutral surfactant Triton X-100, there appears to be some difference in the results of the raw coal and the coal treated with Triton X-100. We believe that the Triton not only binds to the nonpolar sites but also has a strong affinity for the polar sites through electrostatic bonding and interaction between the hydrophobic tails. The addition of molybdenum to coal pretreated with DDAB caused a reduction in the positive charge of the coal surface probably due to possible ionic interaction between the coal surface, the surfactant and the catalyst.

The adsorption isotherm of the coal was characteristic of isotherms for porous samples and the surface area of the coal increased from $30 \mathrm{~m}^{2} / \mathrm{g}$ to $77 \mathrm{~m}^{2} / \mathrm{g}$ when washed with deionized water. This suggests coal washing may be one method of increasing the surface area for surfactant adsorption.

Although the transmission measurements provided valuable information about the coal it resulted in little information on the amount of adsorbed Triton. However, the maximum solid-liquid ratio for optimum surfactant loading of Triton X-100 was determined via the UV-Vis spectrophotometer.

The luminescence intensity measurements showed that the coal and surfactants luminescence weakly. No statistically significant influence was observed from the actions of the surfactants or surfactant-molybdenum catalyst. Qualitative inspection however, showed that SDS might effectively coat coal surfaces and influence catalyst dispersion. Also, catalysts appeared to be better distributed among coal particles and in finer clusters when DDAB and Triton surfactants were used. 


\section{INTRODUCTION}

Zeta potential measurements were conducted to assess the coal surface charge. It has been documented $(1,2)$ that the surface of the bituminous ranked coal is extremely heterogeneous and contains a large number of functional groups. However, the dominant species present on the coal surface are the carboxylic acid and phenolic groups. In aqueous environments the dissociation of the strongly acidic carboxylic acid group is promoted. In the presence of a base, the coal particles will acquire net negative charges due to the removal of a proton from the acidic species. On the contrary, the dissociation of these species in an acidic medium will only protonate the acid, thereby reducing but not eliminating the overall negative charge density. The coal surface particles may become positively charged in extremely acidic media. The type and magnitude of surface charge may be determined from zeta potential measurements. This technique involves suspending coal particles in an aqueous solution contained in a chamber to which a cathode and anode are connected. Upon application of an electric field, a potential is created between the electrodes and the coal particles will migrate towards the anode or cathode depending upon their charge. The zeta potential is proportional to the surface charge and may be displayed by the instrument.

In an effort to examine the adsorption mechanism of the neutral surfactant Triton X-100 to Illinois No. 6 coal a set of experiments were performed. The Illinois No. 6 coal (DECS-24) was obtained from the Penn State Sample Coal Bank. The nonionic surfactant Triton X-100, was obtained from Aldrich Chemical Company Milwaukee, Wisconsin. Pretreatment of the coal surface with various surfactants is one of many methods used to enhance catalyst dispersion for improved liquefaction and gasification. The use of an appropriate surfactant may result in even dispersion of the surfactant on the coal surface thereby creating optimum conditions for catalyst loading by altering the surface hydrophobicity or hydrophilicity.

Prior to the treatment of the coal surface, it must first be characterized to determine possible active sites for the adsorption of surface-active agents. It is well known that the coal surface contains a number of minerals and complex organic species. Coal is extremely heterogeneous, and possesses both hydrophobic and hydrophilic characteristics. Therefore, it is extremely important that the solution conditions such as $\mathrm{pH}$, temperature and concentration are monitored when attempting an adsorption experiment. In addition to the surface adsorption, data regarding the porosity of the coal surface may provide information relating to possible physisorption by the surfactant molecule.

The adsorption of surfactants requires information regarding the surfactant structure and its ability to react with the coal surface. The nonionic character of Triton X-100 must be taken into account when developing a hypothesis to determine possible modes of interaction with the coal surface. The coal surface was first characterized using surface area analysis, and Fourier Transform Infrared Spectroscopy (FTIR). The surface area, which is based upon the BET (Braunner, Emmet and Teller) Theory, provided information regarding the amount of surface area available for possible surface interactions. In addition, based on the isotherm produced, information regarding the porosity of the coal surface was obtained. 
Several IR techniques were used to determine the functionality of the coal surface. Transmission experiments provided general information about the functional groups present. With the use of UV-Vis Spectroscopy an adsorption isotherm based upon the concentration of the surfactant solution before and after its adsorption onto the coal surface was determined. To study the effects of the adsorbance of the surfactant, the DRIFTS (Diffuse Reflectance Infrared Fourier Transform Spectroscopy) technique was used. This technique is much more sensitive to the surface adsorption of species, and may also be used in conjunction with Raman Spectroscopy to provide useful information regarding surface functionality. The experiments performed were preliminary, and it will be interesting to further explore adsorption of Triton X-100 and other surfactants (e.g., SDS and DDAB) by gathering more information regarding the actual orientation of the molecules on the surface of the coal. This may provide more ideas regarding the mechanism for surfactant binding.

Luminescence emission, which can be quantified with an optical microscope and photometer system, offers a measurement capability for establishing the quality of coal surfaces. In a recent investigation of mild coal-surface photo-oxidation, a technique was developed by Mitchell and Davis (3) that allows quantitative measurements of luminescence intensity from the fracture surface for bituminous coals. The work revealed significant variation in emission intensity with degree of oxidation, i.e., intensity decreased with degree of oxidation. This technique was utilized in this study at the Coal and Organic Petrology Labs at Penn State on seven coal specimens and two solid surfactants to assess the quality of the coal surfaces.

\section{EXPERIMENTAL}

\section{Zeta Potential Measurements}

Coal water slurries were prepared by placing 2.5 grams of sample in $500 \mathrm{ml}$ of deionized water containing 0.04 grams of $\mathrm{NaNO}_{3}$ for ionic strength adjustment. To better disperse the particles, the solution was sonicated for twenty minutes using a Branson 2200 Ultrasonic Bath. The coal water slurry was decanted into five flasks each containing $50 \mathrm{ml}$ of solution. After recording the original $\mathrm{pH}$ values, $\mathrm{pH}$ adjustments were made in an effort to reach the desired values (2-12) using $1 \mathrm{M} \mathrm{HCl}$ and $0.5 \mathrm{M} \mathrm{NaOH}$. All pH measurements were made with the aid of a Hanna Instruments Checker $\mathrm{pH}$ meter that had been

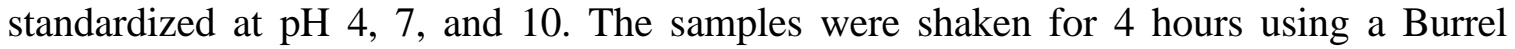
Wrist Action Shaker. After equilibration, the equilibrium $\mathrm{pH}$ value of each sample was recorded. The zeta potential values were measured at room temperature using a Pen Kem Model 501 Zeta Meter.

\section{Surface Area Measurements}

Assessment of the surface area of the coal surface is essential in developing a possible mechanism for surfactant catalyst adsorption. The physisorption of the surfactant is largely dependent upon the surface area available for possible interaction. This area is 
measured by determining the number of nitrogen molecules that are able to form a monolayer on the coal surface. Approximately $0.2 \mathrm{~g}$ of untreated coal was placed in the sample chamber of a Micromeritics ASAP 2000 and degassed to remove any impurities overnight to provide the best possible results. After completion of degassing, the actual sampling began. A known volume of the adsorbate was expelled into the sample chamber and the pressure of the gas was regulated accordingly. A series of measurements were made involving pressure-volume variations until an equilibrium relative pressure was reached. This experiment was repeated using $0.1 \mathrm{~g}$ of coal sample that was previously washed with deionized water.

\section{Transmission Measurements}

Transmission measurements were made using a Nicolet Magna 860 FT-IR spectrometer equipped with a room temperature detector. The pellets used for analysis were prepared using $0.001 \mathrm{~g}$ of coal sample and $0.300 \mathrm{~g}$ of $\mathrm{KBr}$. The mixture was ground and the pellets were pressed using 10,000 tons of pressure for approximately 10 minutes. One hundred and twenty eight interferograms for each spectrum were collected and transformed with a final resolution of $2 \mathrm{~cm}^{-1}$. The time required to collect each spectrum was 139 seconds. Diffuse Reflectance spectra were transformed to Kubelka-Munk Units. UV-Vis spectroscopy measurements were completed using Shimadzu UV-2100 UV-Vis Recording Spectrophotometer. The measurements were taken in absorbance mode using a medium scan speed, and a $1.0 \mathrm{~nm}$ slit width. Ten surfactant solutions were prepared with concentrations ranging from $1.0 \times 10^{-4} \mathrm{M}$ to $5.0 \times 10^{-3} \mathrm{M}$. Eleven coal samples weighing $0.2 \mathrm{~g}$ were measured, and $50 \mathrm{ml}$ of surfactant solution was added to each. To the eleventh water was added to test the effects of washing. The samples were agitated overnight using a Scientific Equipment Products Tube Rotator. The following day, the coal samples were centrifuged at 10,000 rpm for 30 minutes using Sorvall RC SB Plus. The supernatant solution was filtered and analyzed for its surfactant concentration using the UV-Vis Spectrophotometer.

\section{Luminescence Intensity Measurements}

Seven coal specimens and two solid surfactants were prepared and studied. A random subsample was removed from each container and spread evenly onto the level surface of modeling clay affixed to a glass microscope slide. To secure their position, particles were pressed flat into the clay between glass microscope slides in a mounting press. Relatively flat regions of particles were brought into focus in reflected white light using a Leitz MPV-2 research microscope with an air objective at 625 total magnification. Measurement of luminescence intensity was performed by directing 390-490 nm light from a mercury-arc lamp onto exposed coal surfaces. Because the energy absorbed by the specimen is re-admitted (emission) at a longer wavelength, an interference filter was used to eliminate light below $510 \mathrm{~nm}$. $600 \mathrm{~nm}(+/-60 \mathrm{~nm})$ light was allowed to impinge on the photomultiplier. The photoelectric system was standardized with respect to the intensity of a uranyl glass. The procedure was repeated for 20 individual particles and the mean luminescence intensity value and standard deviation were determined (Table 1). 


\section{RESULTS AND DISCUSSION}

\section{Zeta Potential}

The results of the zeta potential measurements provide qualitative information useful for evaluating trends of the surface charge due to the modification of the surface by the adsorption of surfactants and catalyst molecules. Measurements conducted for the parent coal, as illustrated in Figure 1, show that across a broad range of $\mathrm{pH}$ values, the coal has an overall negative surface charge. According to Figures 2-4, the adsorption of surfactants caused a change in the surface charge measurements. The coal, which was treated with the anionic surfactant SDS, maintained an overall net negative surface charge. Interaction between the polar sites of the surfactant and the coal may have caused the reduction in the negativity of the coal surface. The interaction between the coal particles and the cationic surfactant DDAB caused the opposite effect. According to Figure 3, the surface charge became more positive with DDAB. This may be attributed to a combination of the ionic and hydrophobic interactions between the molecules, thereby causing a reduction of the negative surface charge.

Figure 4 depicts the zeta potential results of the parent coal treated with the neutral surfactant, Triton X-100. This figure is interesting, as there should have been virtually no ionic interaction between the coal surface and the surfactant molecule. Thus, we would expect to see a diagram that was exactly like the parent coal. However, although the figures are not identical, it should be noted that Figure 4 is similar to Figure 1 in that it shows an overall negative surface charge throughout the entire $\mathrm{pH}$ range. The trend illustrated in Figure 4 is also largely due to polar interactions between the surfactant molecule and the coal surface.

Triton X-100 is a neutral surfactant that has the ability to bind with a variety of sites on the coal surface. Based on the experiments, zeta potential results have shown that over a wide range of $\mathrm{pH}$ values (2-12), the overall surface charge of the coal water slurry is negative. This should not suggest that there are only anionic bonding sites available on the coal surface. The coal water slurry has a natural $\mathrm{pH}$ of 4 . Therefore, there are a number of strong acidic species available for surfactant binding in solution. Because of the heterogeneity of the surface there exists a variety of possibilities for surfactant binding. Based on literature reviews, it is our supposition that the Triton X-100 molecule not only binds to the non-polar sites on the coal surface but also has a strong affinity for the polar sites. It is also possible that following electrostatic bonding between the molecule and the surface, there may be some interaction between the hydrophobic tails leading to flocculation.

Figure 5 shows the results of the zeta potential of the coal treated with DDAB and the catalyst molybdenum. This figure may easily be compared to Figure 3 that shows the zeta potential of coal treated with only DDAB. It is assumed that the addition of the molybdenum anions caused the reduction in the positive surface charge shown in Figure 3. This is to be expected, due to possible ionic interaction between the coal surface, the surfactant and the catalyst. 


\section{Surface Area}

The adsorption isotherm at the gas-solid interface was established according to the BET method. Because the coal surface is porous, the shape of the isotherm differs from that of a uniform solid surface. According to Figure 6, the adsorption isotherm for the coal surface is characteristic of that of a typical porous solid. The logarithmic nature of the curve is due to the gradual change in the relative pressure of the system needed to fill the variety of pore diameters over a change in adsorbate volume of the system. The "knee" in the curve is formed due to the formation of monolayer coverage of the coal surface. This is followed by the subsequent layering of condensed liquid in the pores due to an increase in the vapor pressure until a saturation point is reached, at which point the system has reached equilibrium. This state of equilibrium can be maintained until a decrease in the pressure of the system begins, which facilitates the desorption process, as illustrated by the desorption curve.

The measured surface area of the coal surface was $30 \mathrm{~m}^{2} / \mathrm{g}$. The same experiment was performed on a sample of coal that was washed with deionized water (Figure 7). The surface area of the coal more than doubled to $77 \mathrm{~m}^{2} / \mathrm{g}$ when washed. This is an indication that many of the species present within the coal sample are water-soluble and may be loosely bond. Since surfactant loading takes place in an aqueous solution, it is possible that many of the polar surface groups may become easily dissociated from the coal surface within the solution. This also presents the possibility that coal washing may be one method of increasing the number of active sites available for nonpolar surfactant adsorption. Further investigation of the surface area of surfactant adsorbed coal species should show a decrease in surface area with an increase in surfactant loading. The greater the decrease, the greater the amount of surface coverage due to surfactant loading. This binding may be due to either electrostatic forces or Van der Walls forces depending on the classification of the surfactant and the amount of surfactant loading.

\section{Transmission Measurements}

The IR transmission spectrum was collected to give an idea about the surface functionality. As evidenced by Figure 8, there appears to be an abundance of hydrocarbon functional groups present that may serve as possible sites for hydrophobic interaction. The aromatic ring stretching is shown at $2918 \mathrm{~cm}^{-1}$ and also $1608 \mathrm{~cm}^{-1}$. The broad peak at $3360 \mathrm{~cm}^{-1}$ may be due to the dissociation of water molecules in the form of a hydroxyl group. In addition to this, there appears to be a significant aliphatic stretching peak present at $2865 \mathrm{~cm}^{-1}$. Although the transmission spectrum provides a great deal of information about the coal, it produces very little results in determining the presence of the adsorbed Triton.

The DRIFTS technique was utilized for its ability to recognize surface adsorbed species. However, we encountered difficulty in distinguishing peaks caused by the surfactant and those caused by the coal, because of the abundance of similar functional groups present. The interference of the coal peaks with the Triton may have been even greater with the use of transmission methods. Further investigation will allow better determination of the surface adsorbed species present. 
To gain knowledge regarding adsorption at the solid/liquid interface, we established the adsorption isotherm. According to the literature, surfactant molecules bind to active sites at low concentrations horizontal to the surface, due to electrostatic interactions. With an increase in surfactant adsorption, their orientation becomes perpendicular to the surface at which point the surface coverage increases until all bonding sites are occupied. As surface coverage increases, the surface charge decreases and tends to zero due to ion pairing. After the formation of a monolayer of surfactant, hydrophobic interaction between the tails of the surfactants may begin to form hemimicelles causing charge reversal. It is at this point that excess surfactant will be expelled into the solution.

With the use of the UV-Vis Spectrophotometer the amount of surfactant adsorbed on the coal surface was measured by testing the difference in the concentration of the surfactant solution loaded and that of the supernatant after loading. According to Figure 9, the greatest amount of Triton adsorption occurs at approximately $147 \mathrm{mg} / \mathrm{g}$ coal which corresponds to an equilibrium concentration of $1.6 \mathrm{mmol} / \mathrm{l}$ of solution. Based upon this experiment, $50 \mathrm{ml}$ of $2.5 \times 10^{-3} \mathrm{M}$ Triton solution is the maximum amount of surfactant solution that can be adsorbed by $0.20 \mathrm{~g}$ of coal to reach maximum saturation. As shown by the curve, the amount of surfactant steadily increases until it reaches its maximum and then there is a logarithmic decline in surfactant after this point.

From this study we have determined that there are a number of sites available for possible surfactant-coal interaction. We expect that there will be some hydrophobic interaction based upon the numerous amounts of hydrocarbon groups that are present. This does not eliminate the possibility for polar interaction. In addition to this, we have determined the maximum solid-liquid ratio for optimum surfactant loading of Triton X100 .

\section{Luminescence Intensity}

Regarding the nature of the surfactants, sodium dodecyl sulfate (SDS) appeared as a transparent/translucent material exhibiting crystal structures when observed in reflected white light. Crystals were sometimes separated or intersected by veins of fine-textured, granular material. SDS had the lowest measured luminescence intensity of all the specimens evaluated (Table 1), perhaps because of its transparent nature which may be responsible for internal re-absorption of the emitted light energy. Dodecyl ethyl dimethyl ammonium bromide (DDAB) appeared to be a wax-like substance that is easily deformed and readily aggregates when attempting to crush. The material exhibits a higher luminescence compared to SDS (Table 1). Under white and blue reflected light, DDAB appeared as formless, rounded particles and aggregates with no crystal edges or cleavage being observed. Luminescence intensity was not measured on the Triton-X.

The measured luminescence intensity of fracture surfaces of the unprepared Illinois \#6 coal was found to be significantly lower $(0.49+/-0.06)$ than was measured a year ago at the Coal and Organic Petrology Labs at Penn State $(0.66+/-0.04)$. This may reflect surface deterioration resulting from extended exposure to air, sunlight or some other laboratory conditions. 
The influence of surfactants on the luminescence properties of the Illinois \#6 coal are shown in Table 1 and depicted graphically in Figure 10. There appears to be a slight intensity increase over the mean of the coal when contacted with the surfactants. However, as demonstrated in the figure, this increase is not statistically significant. Furthermore, there are extenuating circumstances that are not expressed by the numbers.

The SDS surfactant was observed mostly in direct contact with coal surfaces, but some was seen surrounding coal particles as well as independent crystals not associated with coal particles. Many of the higher intensity readings taken were obtained from coated areas with the coal surface clearly visible through the coating. On the other hand, the DDAB surfactant commonly was observed as separate particles either not associated with coal surfaces or attached at a single point. One very high intensity reading was taken from an irregular depression on a coal surface that is assumed to contain the DDAB surfactant. Regarding the Triton surfactant, none could be visibly identified on the surface and, other than showing a slightly higher mean intensity, no other differences were observed compared to the unprepared coal sample.

Dispersion of the molybdenum catalyst should not have much influence on the luminescence intensity other than obscuring the coal surface and for the most part this is confirmed by the data (Triton and DDAB, Figure 10). This of course is provided that no carrier solvents were used to dissolve or distribute the catalyst. However, a fairly large decrease in mean intensity was measured for the specimen containing SDS and catalyst that can not be explained. Observation of this specimen under white and blue light revealed SDS surrounding and coating particles in the manner previously described. Presumably, the molybdenum catalyst can be observed in the specimen in two forms: small $(5-10 \mu \mathrm{m})$ clusters of dark iridescent particles that are observed attached sporadically to the coal surface or in direct contact with SDS, and catalyst that is also found as independent clusters $(10-20 \mu \mathrm{m})$ associated with SDS. In no area was a uniform even coating of catalyst or SDS and catalyst seen associated with coal surfaces.

Inspection of specimens containing Triton and DDAB in association with the molybdenum catalyst did not reveal much of the surfactant. However, in both cases the clusters of catalyst were small $(5-10 \mu \mathrm{m})$ and; qualitatively, appeared to be more uniformly distributed on the coal surface. This does not mean that there was an even uniform coating, just that most every coal particle had some associated catalyst.

Briefly, from the series of specimens studied, luminescence intensity measurements show that the coal and solid surfactants luminescence weakly. Also, no statistically significant influence was observed that resulted from the action of the surfactants or surfactant-molybdenum catalyst. Qualitative inspection of the natural fracture surfaces of these treated coals, however, shows that the SDS surfactant may effectively coat coal surfaces and may influence the dispersion of catalyst particles. Catalyst appeared to be better distributed among coal particles and in finer clusters when the DDAB and Triton surfactants were employed. 


\section{REFERENCES}

1. Abotsi, G., 1996, Interfacial Properties of Coal: A Guide to Catalyst Loading and Dispersion for Coal Conversion, Energeia, Center for Applied Energy Research, University of Kentucky, Vol. 7, No. 5, pp1-3

2. Owen, M. J., 1989, Enhancement of Mechanical Dewatering of Fine Coal by SurfaceActive Additives, In Interfacial Phenomena in Coal Technology, G . D. Botsaris and Y. M. Gazman, Editors, Marcel Dekker, Inc., NY, NY, pp 157-195

3. Mitchell, G.D. and Davis, A., 1997, Surface Properties of Photo-Oxidized Bituminous Coals, Final Report, September 1, 1993 to February 28, 1997, DOE Grant No. DE-FE2293PC93223, August 1997.

Table 1 Mean Luminescence Intensity at $600 \mathrm{~nm}$

\begin{tabular}{lccc}
\hline Sample Id. & Luminexcence Intnesity, & Stf. Dev. & $\pm 95 \%$ Confidence \\
& $\mathrm{F}_{\mathrm{I} 600}$ & & \\
\hline SDS - Surfactant & 0.287 & 0.093 & 0.042 \\
DDAB - Surfactant & 0.512 & 0.282 & 0.129 \\
\#1 Illinois \#6 (DECS-24) & 0.487 & 0.125 & 0.056 \\
\#2 coal w/0.1M SDS & 0.546 & 0.135 & 0.061 \\
\#6 Coal w/0.1M SDS \& & 0.443 & 0.079 & 0.036 \\
Mo Cat. & & & \\
\#3 Coal w/0.1M DDAB & 0.555 & 0.197 & 0.080 \\
\#7 Coal w/0.1M DDAB \& & 0.569 & 0.127 & 0.057 \\
Mo Cat. & & & \\
\#4 Coal w/0.1M Triton & 0.527 & 0.087 & 0.039 \\
\#5 Coal w/0.1M Triton \& & 0.523 & 0.112 & 0.050 \\
Mo Cat. & & & \\
\hline
\end{tabular}




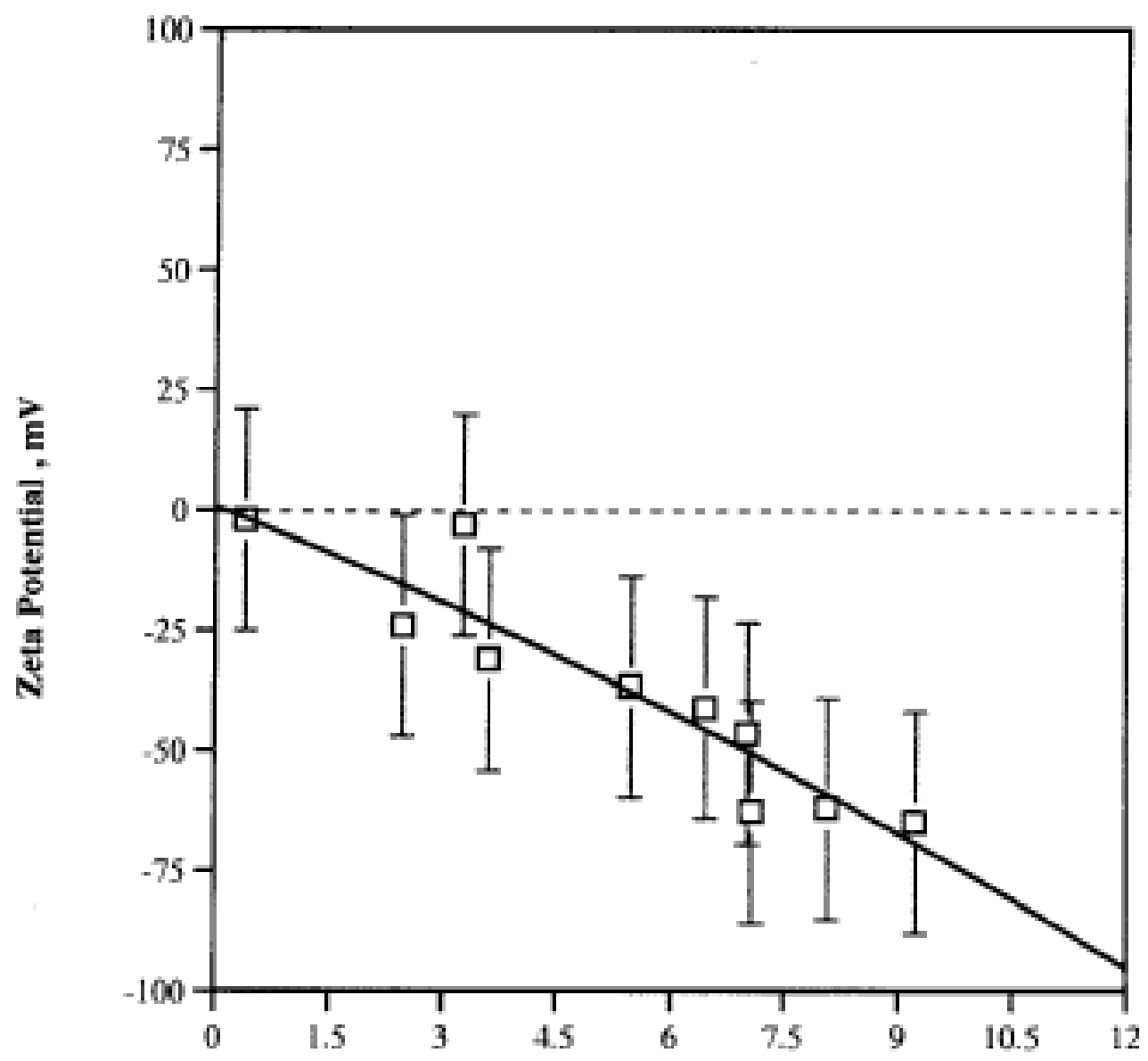

Equilibrium pH

Figure 1 Zeta potential of the parent coal. 


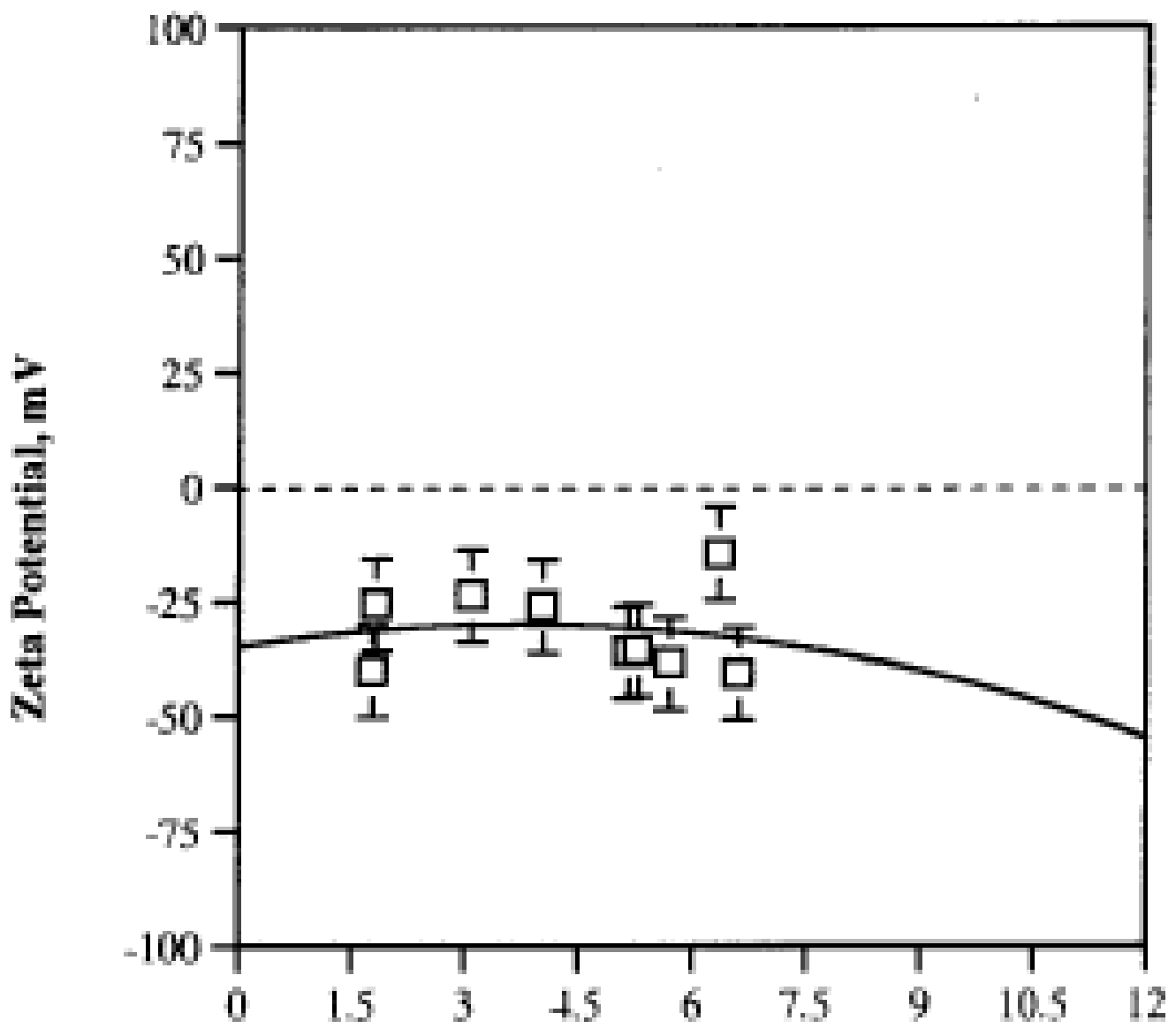

Equilibrium pH

Figure 2 Zeta potential of coal treated with SDS. 


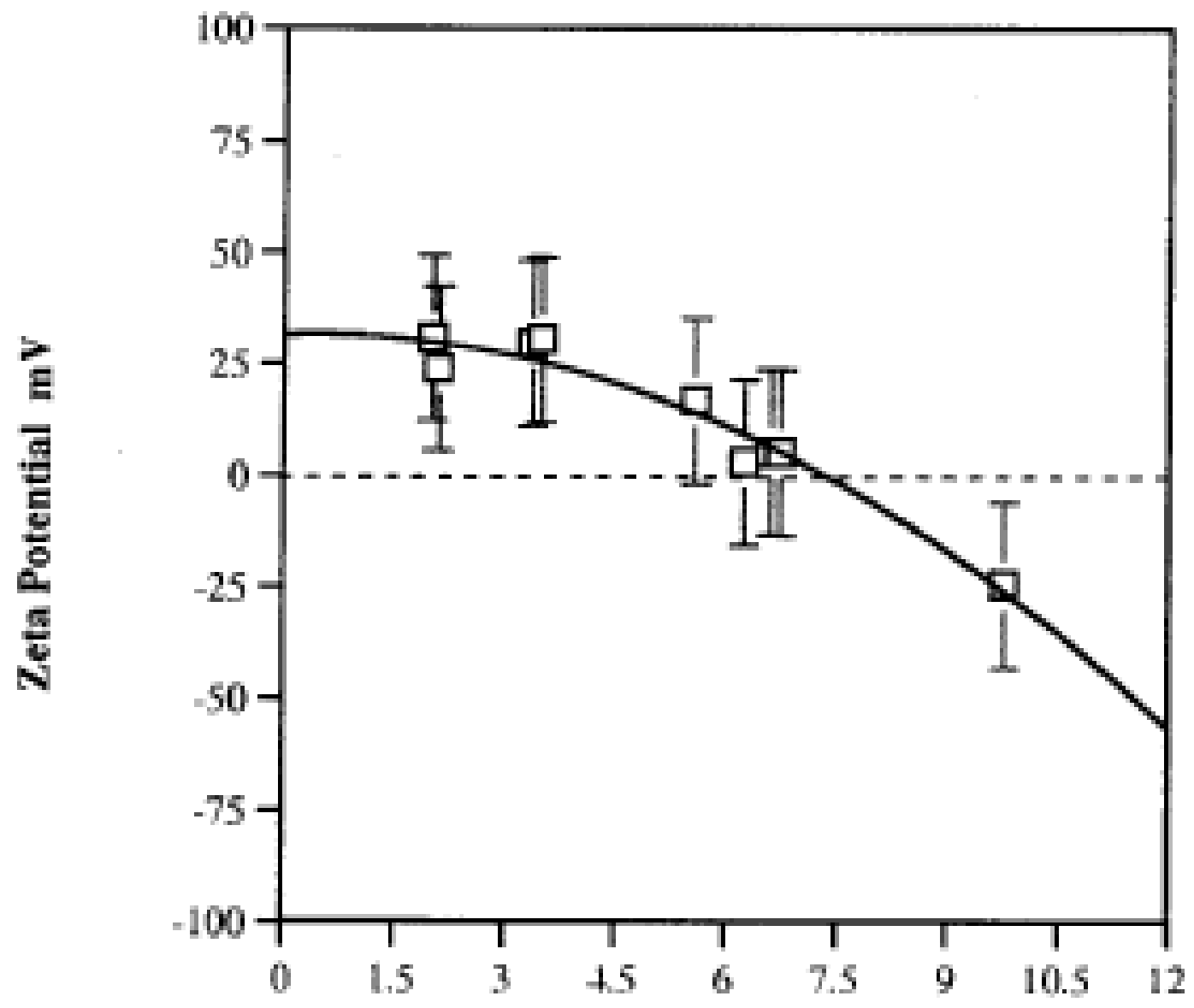

Equilibrium oH

Figure 3 Zeta potential of coal treated with DDAB. 


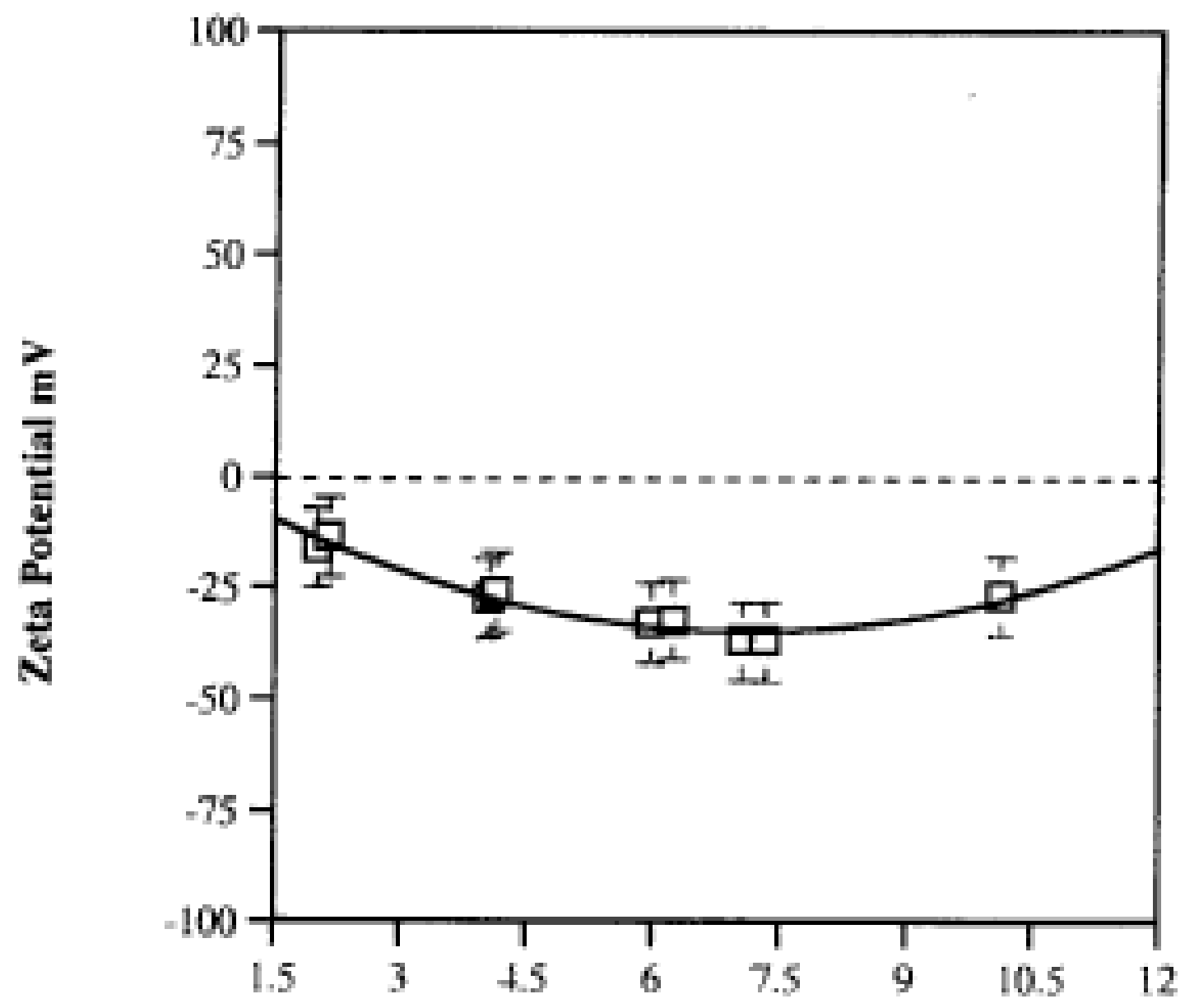

Equilibrium $\mathbf{p H}$

Figure 4 Zeta potential of coal treated with Triton X-100. 


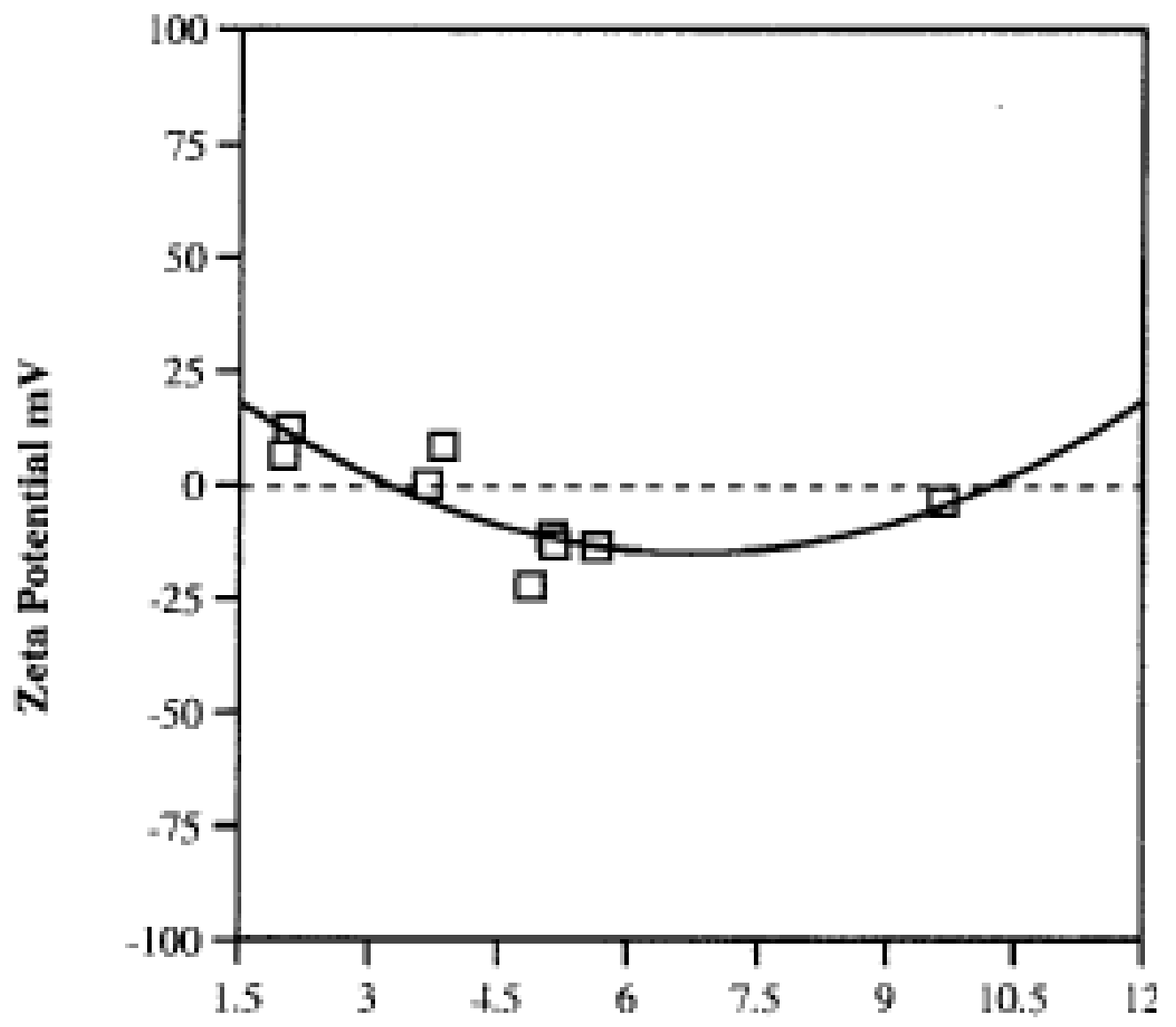

Equilibrium pH

Figure 5 Zeta potential of coal + DDAB + Mo catalyst. 


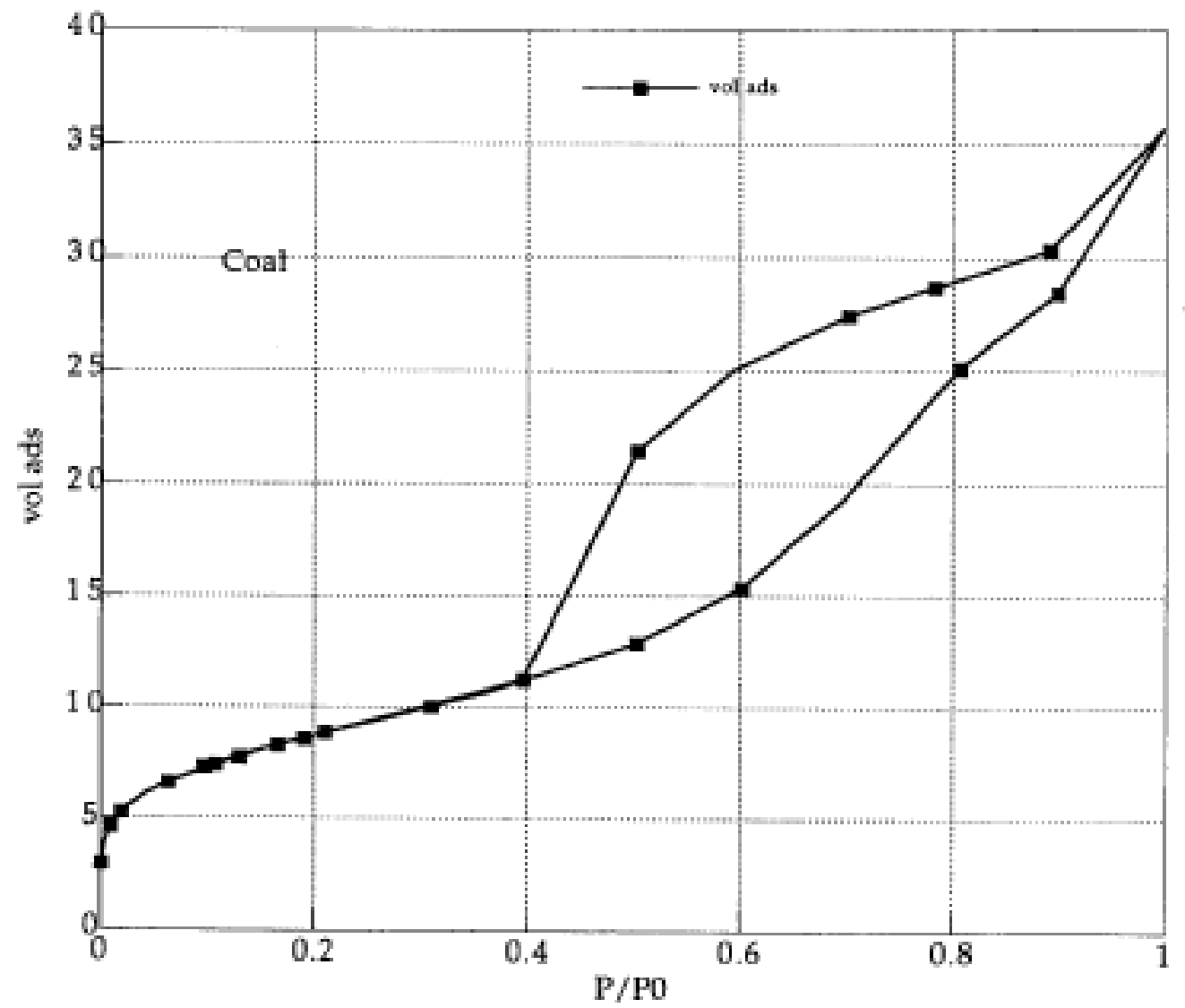

Figure 6 Adsorption isotherm of the parent coal 


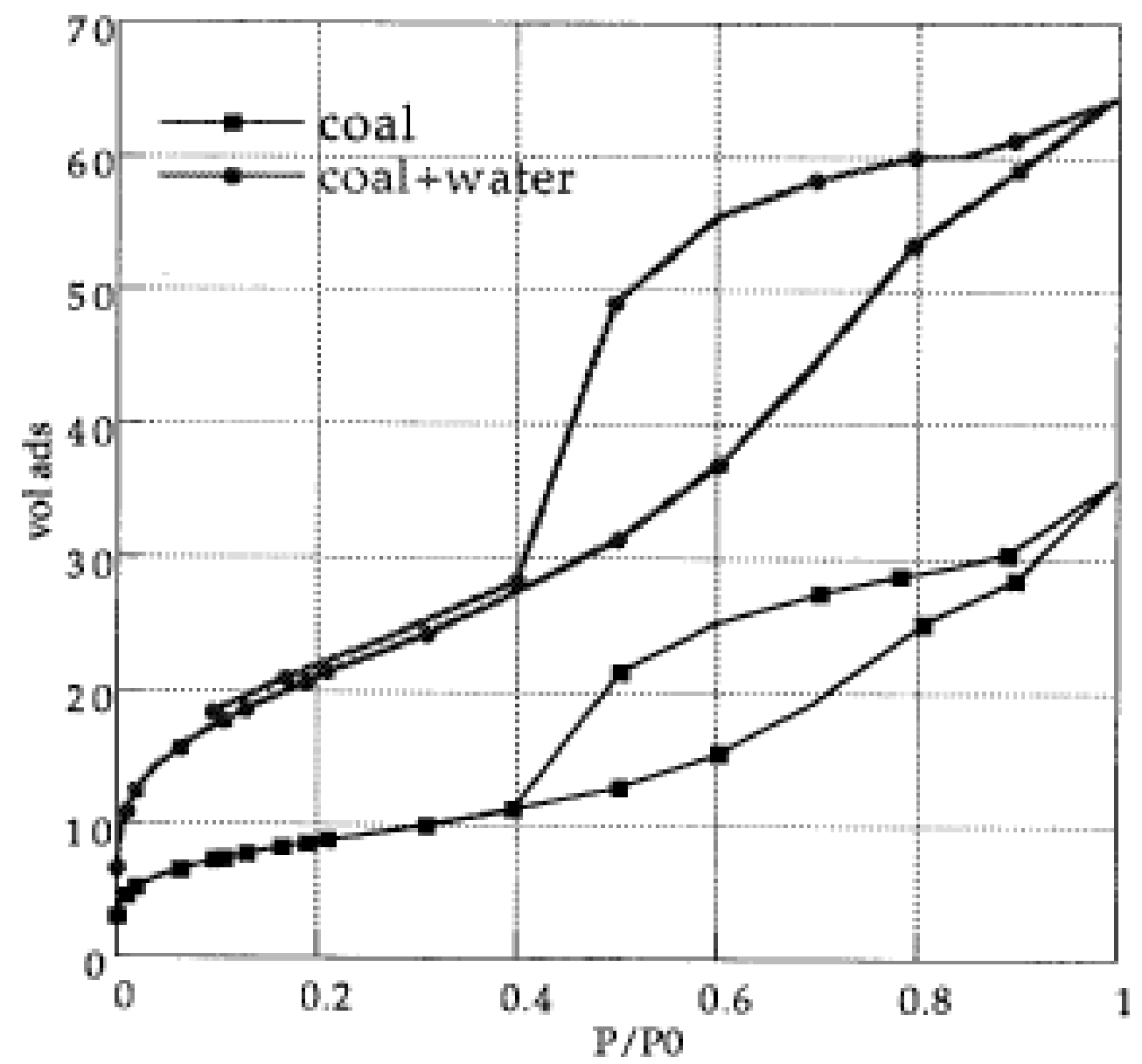

Figure 7. Adsorption isotherms for coal and coal $+\mathrm{H} 2 \mathrm{O}$ 


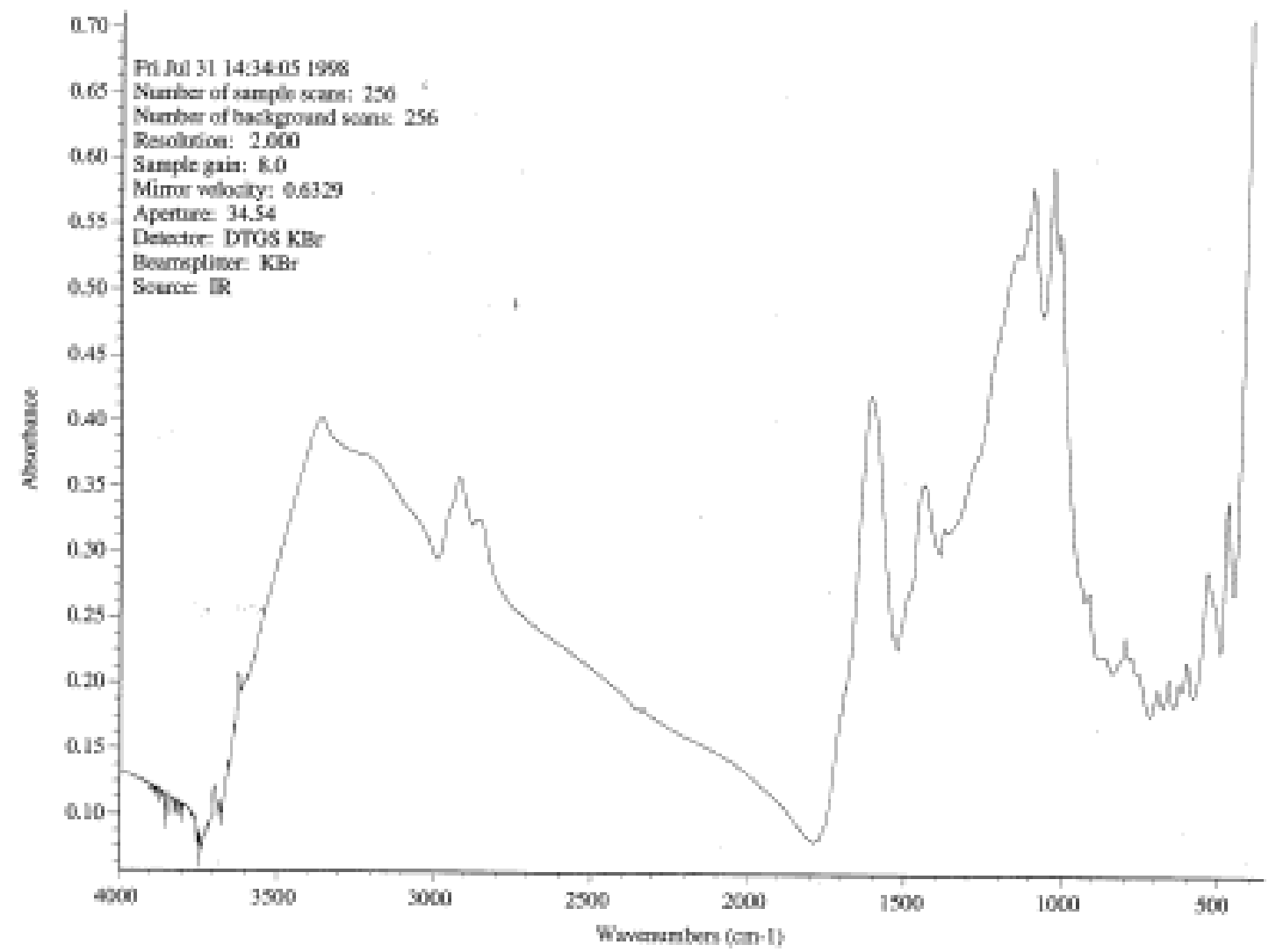

Figure 8. IR Transmission Spectrum of parent coal 


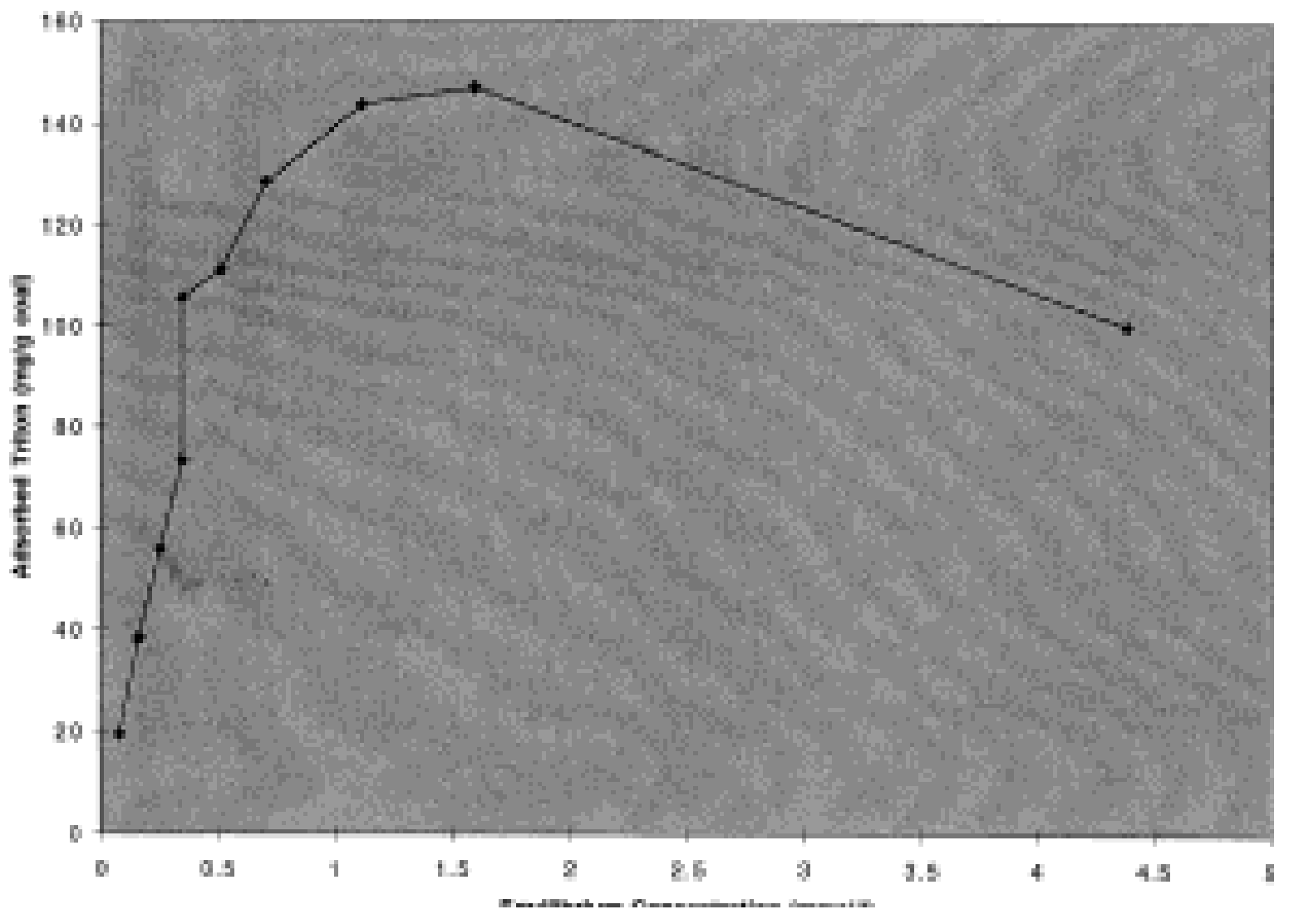

Figure 9 Triton adsorption isotherm 


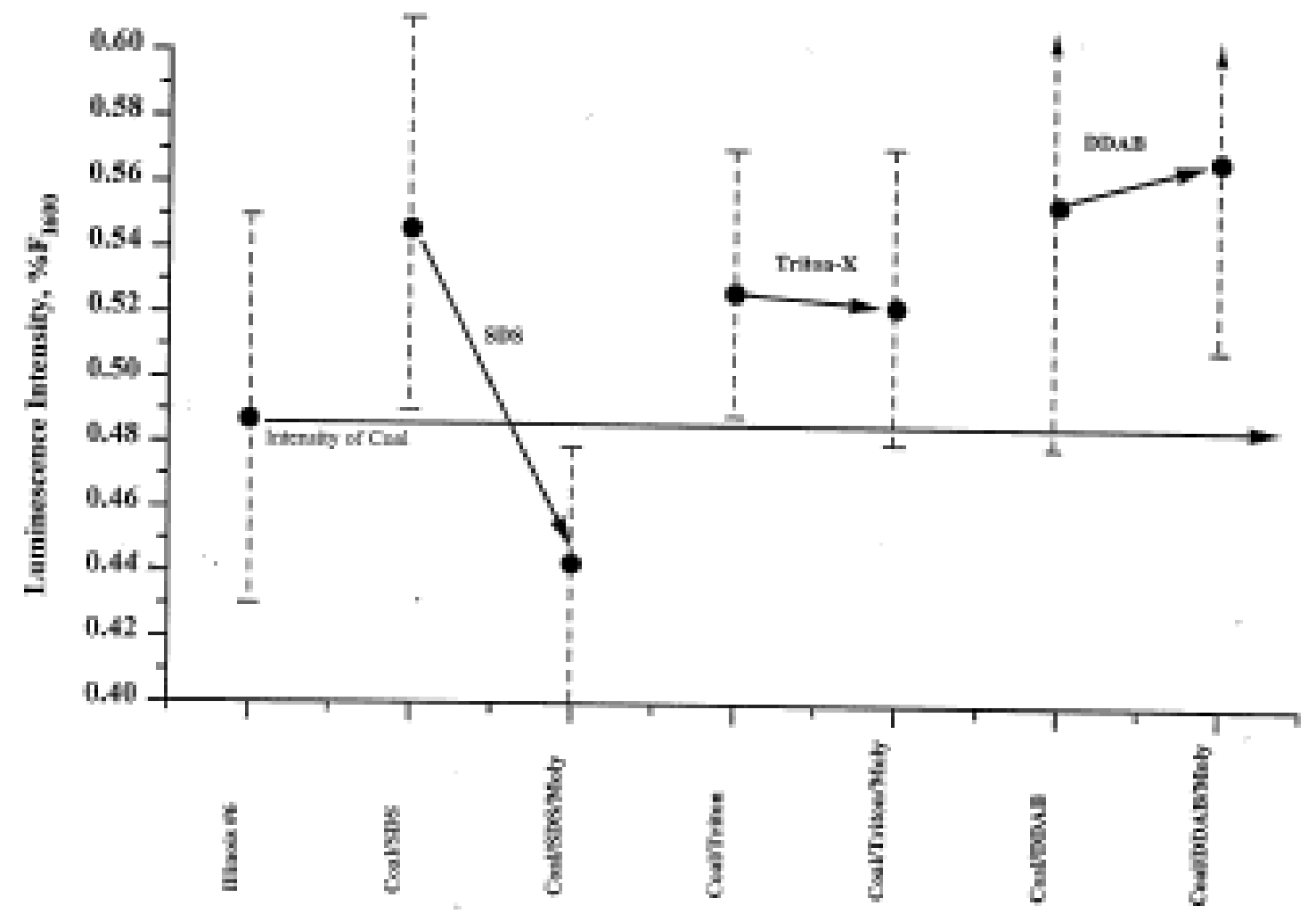

Figure 10 Variation in luminescence intensity resulting from the presence of surfactant and molybdenum catalyst. 